\title{
Identification and characterization of long non-coding RNAs in subcutaneous adipose tissue from castrated and intact full-sib pair Huainan male pigs
}

Jing Wang ${ }^{1 \dagger}$, Liushuai Hua ${ }^{1 \dagger}$, Junfeng Chen ${ }^{1}$, Jiaqing Zhang ${ }^{1}$, Xianxiao Bai ${ }^{1}$, Binwen Gao ${ }^{1}$, Congjun Li ${ }^{2}$, Zhihai Shi ${ }^{1}$, Weidong Sheng ${ }^{1}$, Yuan $\mathrm{Gao}^{3}$ and Baosong Xing ${ }^{1 *}$

\begin{abstract}
Background: Long non-coding RNAs (IncRNAs) regulate adipose tissue metabolism, however, their function on testosterone deficiency related obesity in humans is less understood. For this research, intact and castrated male pigs are the best model animal because of their similar proportional organ sizes, cardiovascular systems and metabolic features.

Results: We identified IncRNAs in subcutaneous adipose tissue by deep RNA-sequencing using the intact and castrated Huainan male pigs. The results showed that castration reduced serum testosterone but increased body fatness-related traits (serum triglyceride levels, backfat thickness, intramuscular fat content, and adipocyte size). Meanwhile, 343 IncRNAs from subcutaneous adipose tissue were identified, including 223 intergenic IncRNAs (lincRNAs), 68 anti-sense IncRNAs, and 52 intronic IncRNAs. It was predicted that there were 416 recognition sites for C/EBPa in the 303 IncRNA promoter region, and 13 adipogenesis-promoting miRNAs and five adipogenesisdepressing miRNAs target these IncRNAs. Eighteen IncRNAs, including nine up- and nine down-regulated had more than 2-fold differential expression between the castrated and intact male pigs ( $q$-value $<0.05$ ). Functional analysis indicated that these 18 IncRNAs and their target genes were involved in fatty acid, insulin, and the adipocytokine signaling pathway. We further analyzed the features of a conserved mouse IncRNA gene ENSMUST00000189966 and found it mainly expressed in the cell nucleus and target the Nuclear Receptor Subfamily 2 Group F Member 2 (NR2F2) gene. In 3 T3-L1 cells, differentiation down-regulated their expression, but dihydrotestosterone (DHT) significantly up-regulated their expression in a concentration-dependent manner $(P<0.05)$.
\end{abstract}

Conclusions: These results suggested that IncRNAs and their target genes might participated in the castrationinduced fat deposition and provide a new therapeutic target for combatting testosterone deficiency-related obesity.

Keywords: Subcutaneous adipose tissue, LncRNA, Pig, Fat deposition

\footnotetext{
* Correspondence: baosong008@126.com

'Equal contributors

'Henan Key Laboratory of Farm Animal Breeding and Nutritional Regulation,

Institute of Animal Husbandry and Veterinary Science, Henan Academy of

Agricultural Sciences, No.116 Huayuan road, Zhengzhou 450002, People's

Republic of China

Full list of author information is available at the end of the article
}

(c) The Author(s). 2017 Open Access This article is distributed under the terms of the Creative Commons Attribution 4.0 International License (http://creativecommons.org/licenses/by/4.0/), which permits unrestricted use, distribution, and reproduction in any medium, provided you give appropriate credit to the original author(s) and the source, provide a link to the Creative Commons license, and indicate if changes were made. The Creative Commons Public Domain Dedication waiver (http://creativecommons.org/publicdomain/zero/1.0/) applies to the data made available in this article, unless otherwise stated. 


\section{Background}

Sex glands not only play a regulatory role in reproductive traits, but also in growth traits [1]. Researchers have found that gonadal steroid hormones like testosterone could promote lipolysis and releasing energy. In the process of aging or illness, reduced levels of androgens are associated with obesity (particularly in visceral fat) and related diseases in human [2]. The castrated male pigs, with the similar organ size, metabolic features, cardiovascular systems, are the best animal model for researching human testosterone deficiency-related diseases [3-5].

Long noncoding RNAs (lncRNAs) are transcripts that structurally resemble mRNAs but do not encode proteins. LncRNAs regulate gene expression at the posttranscriptional level and play important roles in numerous disease and physiological metabolism processes, including $\mathrm{X}$-chromosome inactivation [6], embryonic development [7], pluripotency maintenance [8]. Recently, a growing number of reports has demonstrated that lncRNAs regulated adipogenesis by activating or silencing key genes through recruiting chromatin modification complexes or acting as competing endogenous RNAs (ceRNAs): ADINR (adipogenic differentiation induced noncoding RNA), promoted adipogenesis by activating CCAAT/enhancer binding protein alpha $(\mathrm{C} / \mathrm{EBP} \alpha)$ transcription in cis [9]. LncRNA-AK038898 (Blnc1) promoted brown adipocyte differentiation and stimulated the thermogenic phenotype by forming a complex and feedforward regulatory loop with transcription factor EBF2 [10]. PU.1 (also known as SPI1, spleen focus forming virus (SFFV) proviral integration oncogene) inhibited adipogenesis by the downregulation of adipogenic master gene PPARY [11], and its antisense long non-coding RNAs promotes adipogenesis by preventing its mRNA translation [12]. LncRNA ADNCR (adipocyte differentiation-associated long noncoding RNA) suppressed adipocyte differentiation by function as a ceRNA for miR-204, whose target geneSIRT1 was known to repress PPAR $\gamma$ activity and inhibit adipocyte differentiation [13].

The regulatory function of IncRNA on fat metabolism suggests that it is feasible to research the testosterone deficiency-related fat deposition from the angle of lncRNA. Therefore, we investigated the changes of lncRNA expression in the subcutaneous adipose tissue (SAD) between intact and castrated male pigs, and this provides a novel view of the role of IncRNAs in testosterone deficiency-induced fat deposition as well as basic prophylactic-therapeutic measures for human sex hormone deficiency-related obesity.

\section{Results}

Body fatness traits

In this research, castrated and intact male pigs had similar birth, weaning, and carcass weights $(P>0.05)$. However, the leaf fat weight (nine times greater, $P=0.007$ ), fat percentage $(P=0.020)$, and $6-7$ th rib fat (five times thicker, $P=0.001)$ in castrated male pigs were significantly different with that in intact male pigs (Table 1). Castration resulted in a significant increase in intramuscular fat content in male pigs' longissimus dorsi muscle (LDM, $P<0.01$ ) and psoas major muscle (PMM, $P<0.05$, Table 1$)$.

Castration significantly reduced the serum testosterone level $(P<0.01)$. However, the serum triglyceride (TG) was up-regulated by castration $(P<0.01$, Table 1$)$. These results corresponded to the larger adipocyte size $(P<0.01)$ observed in castrated group compared with intact group (Fig. 1).

\section{Read mapping and transcript assembly}

The redundant and low-quality reads were removed, and a total of $11.60 \mathrm{G}$ and $10.84 \mathrm{G}$ clean bases were obtained in castrated and intact male pigs, respectively. The clean reads were aligned to the reference genome for assembly. Approximately, the mapping rate of the total clean reads to the Sus scrofa genome assembly 10.2 were $81.31 \%$ (castrated male pigs) and $82.48 \%$ (intact male pigs), respectively.

\section{Genomic information of porcine IncRNAs}

In this research, 343 lncRNA genes were found distributed in all chromosomes (Additional file 1: Text file containing identified lncRNA sequence), including 223 intergenic lncRNAs (lincRNAs), 68 anti-sense lncRNAs, and 52 intronic lncRNAs. These 343 lncRNA genes corresponding to 402 transcripts, about 1.2 isoforms per lncRNA locus on average. The size of porcine lncRNAs ranged from 203 to 21,530 nucleotides, the average size was approximately $1520 \mathrm{bp}$. The average exon number of these IncRNA was 2.1 on average $(90.1 \%$ of the total lncRNAs has two exons), the same as that of human lncRNAs [14]. The average length of lncRNAs open reading frame (ORF) was $110 \mathrm{bp}$. We found that $73.5 \%$ of the total lncRNA had target genes (protein-coding genes located in $100 \mathrm{~kb}$ around the lncRNAs), containing 3.4 isoforms per lncRNA locus on average, respectively.

In this research the mean fragments per kilobase of exons per million fragments mapped (FPKM) value of these lncRNAs was 17, and $91.2 \%$ of the lncRNAs with FPKM values less than 15 [15], indicating that the expression levels of the majority of porcine lncRNAs were low, which was similar to the expression levels of human lncRNAs [16]. In this research, we found 562 recognition sites for $\mathrm{C} / \mathrm{EBP} \alpha$ in the 300 identified lncRNAs promoter region, containing $1.9 \mathrm{C} / \mathrm{EBP} \alpha$ recognition site per lncRNA on average (Additional file 2: Table S1). The relationship between the 343 lncRNAs with 18 adipogenesisrelated miRNAs was shown in Table S2 (Additional file 3). We found 13 adipogenesis-promoting miRNAs (let- 


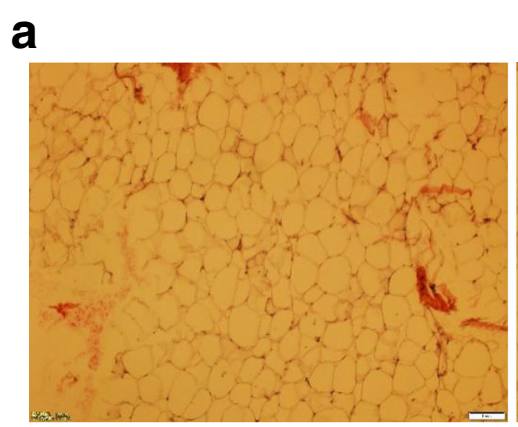

b

$$
\text { Intact male pigs }
$$

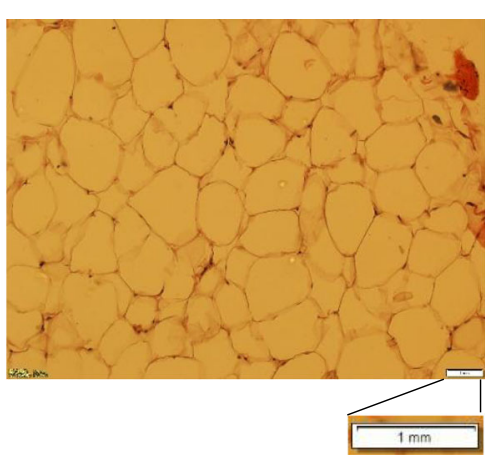

Castrated male pigs

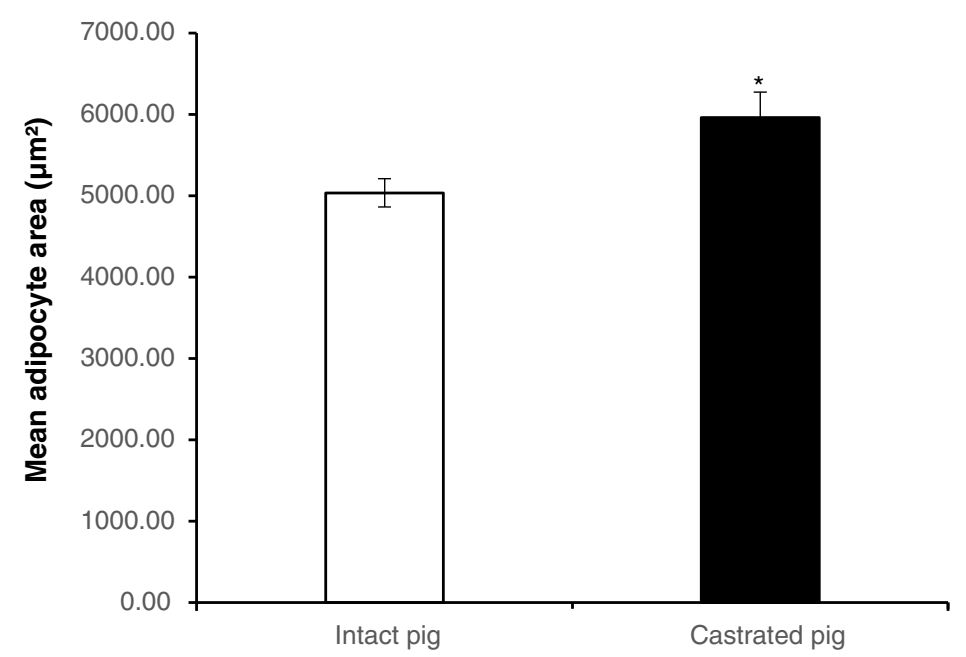

Fig. 1 Histological sections of subcutaneous adipose tissue from intact and castrated male pigs. a H\&E staining of subcutaneous adipose tissue from intact and castrated male pigs (shown at $\times 200$ magnification). $\mathbf{b}$ The mean adipocyte size in intact and castrated male pigs. The adipocyte area was measured using Image J software from three different animals per group. ${ }^{*} P<0.05$

7、 miR-9、miR-15a、miR-17、miR-21、 miR-24、 miR30、 miR-103、 miR-107、 miR-125b、miR-204、 miR210、 and miR-378) target 860 lncRNA loci. Five depressing-adipogenesis miRNAs (miR-27, miR-150, miR221, miR-222, and miR-326) target 217 lncRNAs.

\section{The difference between IncRNAs with protein-coding genes}

We also obtained 12,372 protein-coding genes, with 1.2 isoforms on average $(38,219$ transcripts in total). These protein-coding genes had 518 bp ORF length and 9.8 exons on average, larger than that of the lncRNA genes (Additional file 4: Figure S1a, b). There was only $6.24 \%$ of protein-coding transcripts with two exons, far less than that of lncRNA genes (Additional file 4: Figure S1c).

\section{Differentially expressed IncRNAs in SAD between castrated and intact male pigs}

Eighteen lncRNAs (including nine up- and nine downregulated, Additional file 5: Table S3) and 2645 mRNA (including 1365 up- and 1280 down-regulated, Additional file 6: Table S4), were found to differentially express in SAD between the castrated and intact male pigs ( $q$-value $<0.05$, Fig. 2a). The chromosome distribution shows these mRNAs and lncRNAs location in all of the chromosomes (Additional file 7: Figure S2b).

To validate the sequencing data, eight lncRNAs (four up- and four down-regulated) were randomly selected, and their expression were confirmed via the qRT-PCR method. We calculated the correlation efficiency of qRTPCR and RNA-sequencing data using SPSS software (version 1.70), and the result supported our sequence data $(R=0.882$, Additional file 7 : Figure $S 2)$.

\section{KEGG analysis of the target genes of 18 IncRNAs and the differently expressed protein-coding genes}

According to KEGG functional annotations, the predicted target genes of nine up- and nine downregulated lncRNAs were further classified to identify 


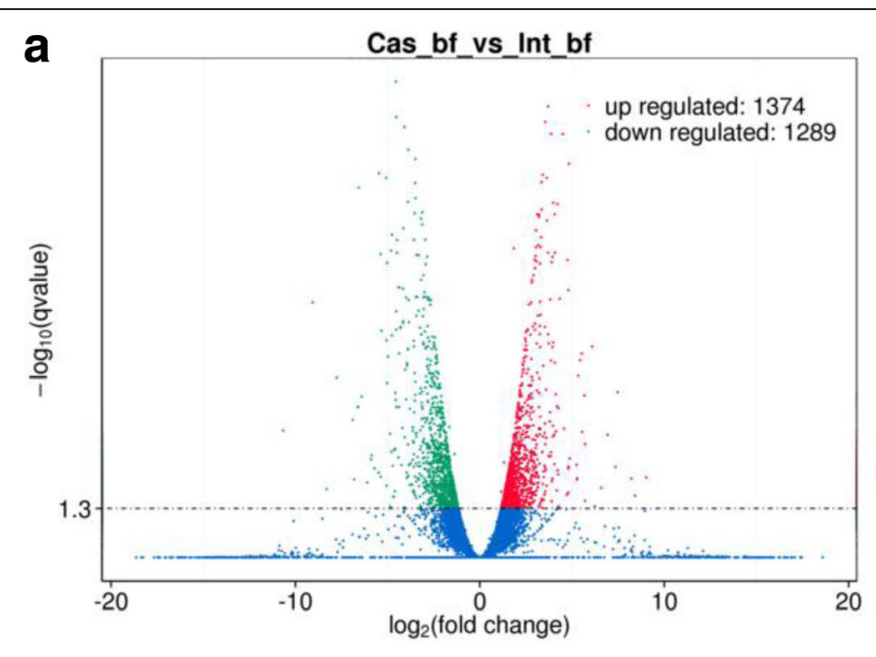

b

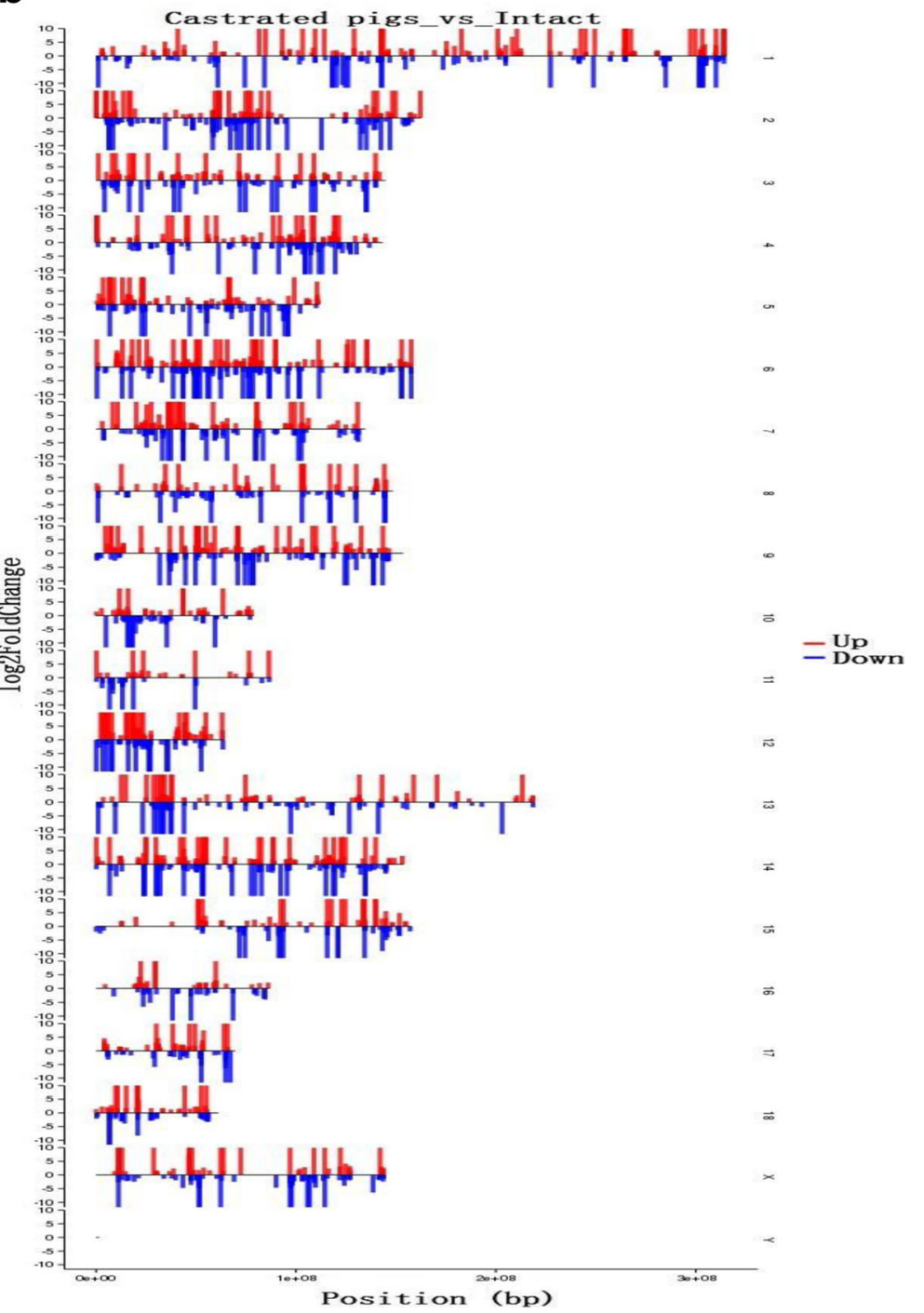

Fig. 2 (See legend on next page.) 
(See figure on previous page.)

Fig. 2 The differentially expressed IncRNAs and mRNA between castrated and intact male pigs and their distribution. a The differentially expressed IncRNAs and mRNA between castrated and intact male pigs. Each point in the figure represents a IncRNA or mRNA. The X axis show the position of IncRNAs and mRNA in different chromosomes, the $Y$ axis show the $\log _{2}$ FoldChange. The red points represent up-regulated IncRNAs or mRNA. The green points represent down-regulated IncRNAs or mRNA. The blue points represent equally expressed IncRNAs or mRNA. b The distribution of the differentially expressed IncRNAs and mRNA. The $Y$ axis show $\log _{2}$ normalized read counts. The red bars represent upregulated IncRNAs or mRNA. The green bars represent down-regulated IncRNAs or mRNA

pathways, and 19 pathways are shown in Fig. 3. It was worth noting that the up-regulated target genes of the majority of lncRNAs in the castrated pigs belonged to the adipocytokine, insulin, fatty acid metabolism, and fatty acid biosynthesis signaling pathways, which were closely related to lipogenesis [17-19]. Another pathway targeted by up-regulated protein-coding genes and lncRNAs is the AMPK signaling pathway (Fig. 4), which is known to be involved in increasing the adipocyte volume and number [20]. Acetyl-CoA carboxylase beta (ACACA) and insulin receptor substrate 1 (IRS1) were the predicted target genes for lncRNAs TCONS_00392681 and TCONS_01199263, respectively. They all upregulated by castration, which indicated that these protein-coding genes and lncRNAs participated in the castration induced lipid deposition by increasing the adipocyte volume and number.

\section{Characterization of ENSMUST00000189966}

Nuclear Receptor Subfamily 2 Group F Member 2 (NR2F2, also known as COUP-TF II) played an important role in the regulation of both hormone and lipid [21]. So TCONS_01646544, one of the downregulated lncRNAs and target NR2F2, was chosen to validate the identified lncRNAs' response to dihydrotestosterone (DHT) and differentiation. To raise efficiency, ENSMUST0000018 9966, a TCONS_01646544 homologous in mice, was used in further research with the 3 T3-L1 cell line.

ENSMUST00000189966 and NR2F2 reached a minimum expressional level on day 2 of differentiation and then gradually rose. On day 8 , the $N R 2 F 2$ expression level was still lower than that on day 0; however, the expression of ENSMUST00000189966 on day 8 was much higher than that on day $0(P<0.01$, Fig. 5a). We found that ENSMUST00000189966 was mainly expressed in nucleus of the 3 T3-L1 cells (Fig. 5b).

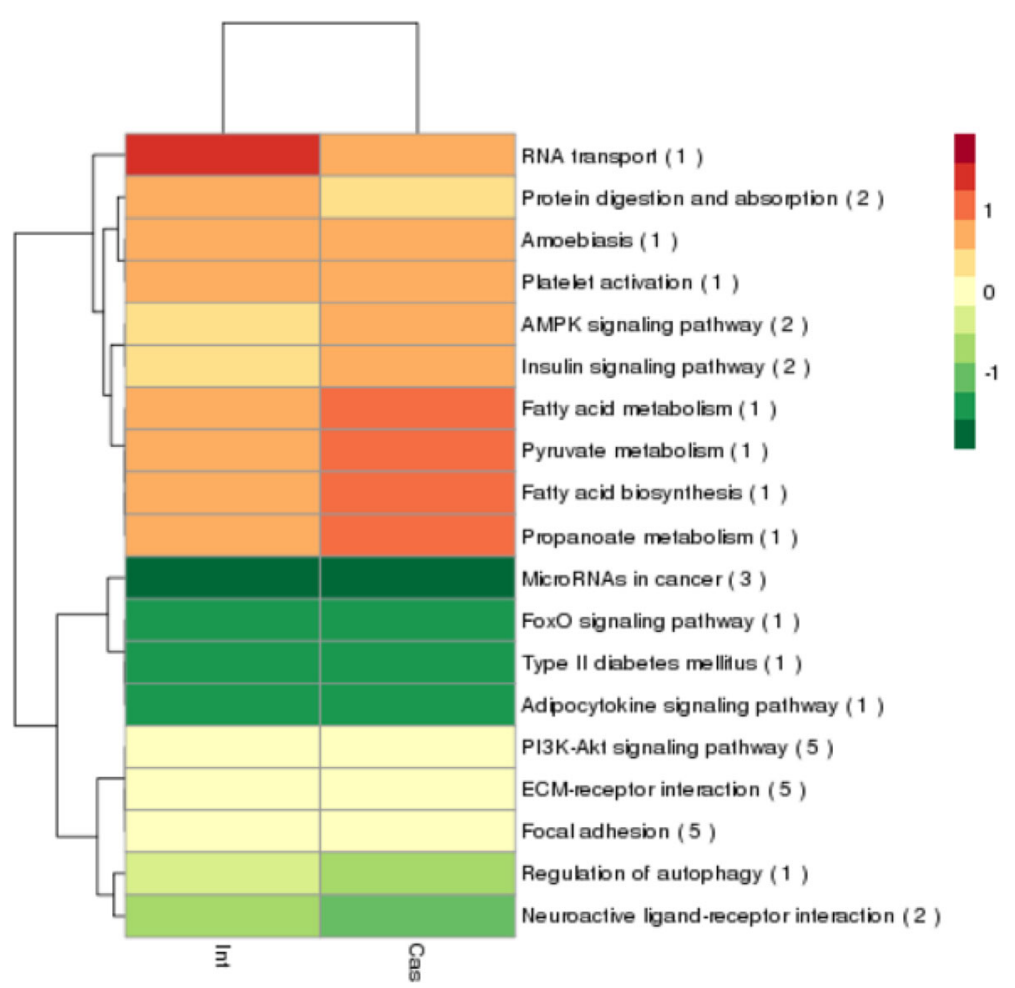

Fig. 3 KEGG pathways enriched for the closely neighboring protein-coding genes of differently expressional IncRNAs in the subcutaneous adipose of castrated and intact male pigs. Green and red represent lower-than-average and higher-than-average signal levels, respectively. The numbers behind the pathway name represent the numbers of differentially expressed genes in that particular pathway. Int: intact male pig, Cas: castrated male pig 


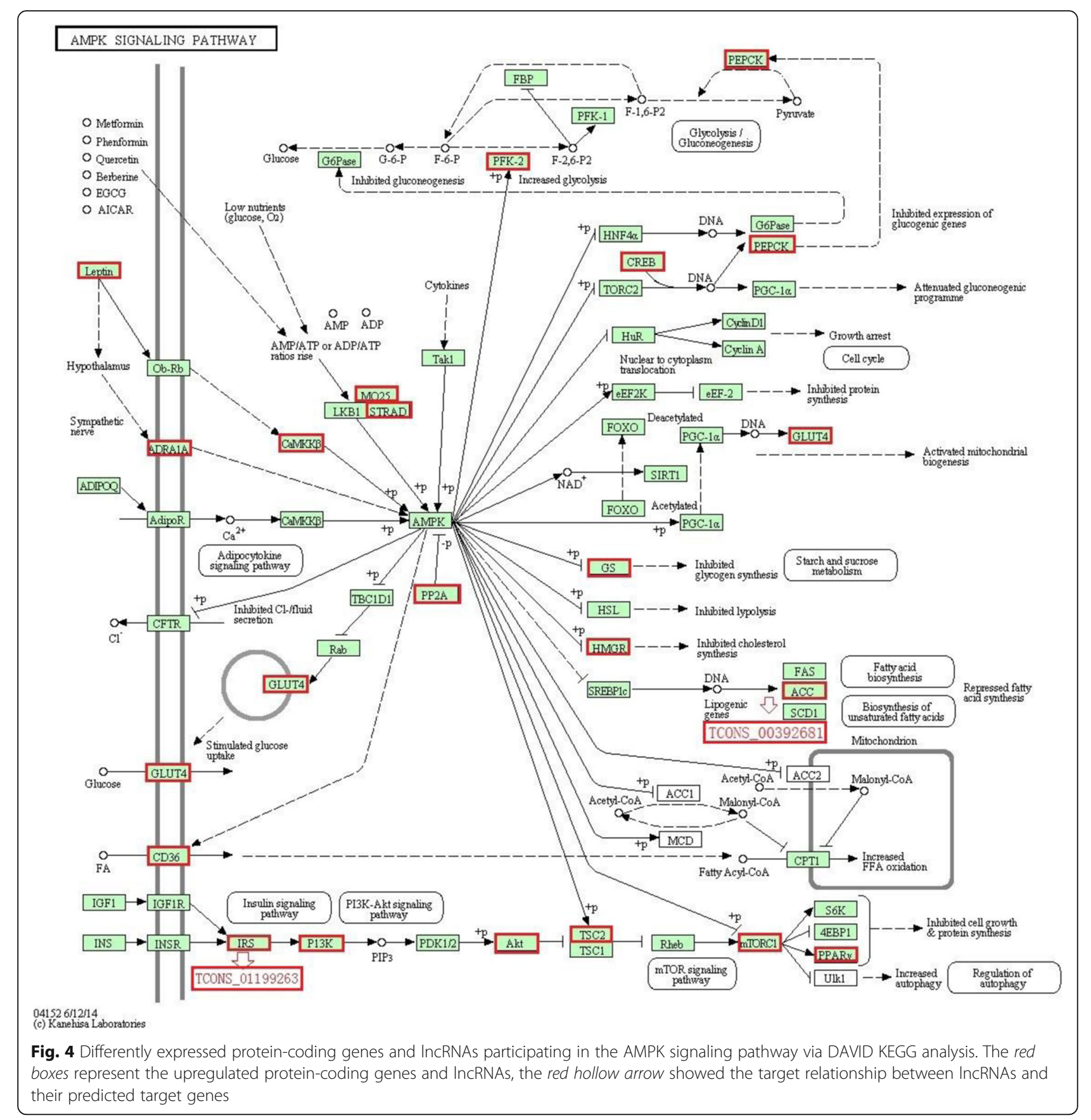

On day 14 of differentiation, Oil-red $\mathrm{O}$ staining was used to confirm the maturity of the 3 T3-L1 cells, then DHT treatment was started. DHT significantly upregulated ENSMUST00000189966 $(P<0.05)$ and NR2F2 $(P<0.01)$ expression in a concentration-dependent manner (Fig. 5c).

\section{Discussion}

In humans, testosterone deficiency causes obesity and associated diseases [15]. Researchers have identified its molecular mechanism from genome and transcriptome angles, using castrated male pigs as the model animal $[22,23]$. However, it remains unclear whether testosterone deficiency can influence lncRNA expression patterns in adipose tissue. In this research, we first combined body fatness traits, serum testosterone and TG levels, and histochemistry analysis with lncRNA sequencing to clarify the molecular mechanism underlying the increased body fatness response to testosterone deficiency.

Several recent studies reported that lncRNAs were directly regulated by key transcription factors that drive cellular differentiation $[24,25]$. In this research, we 
a

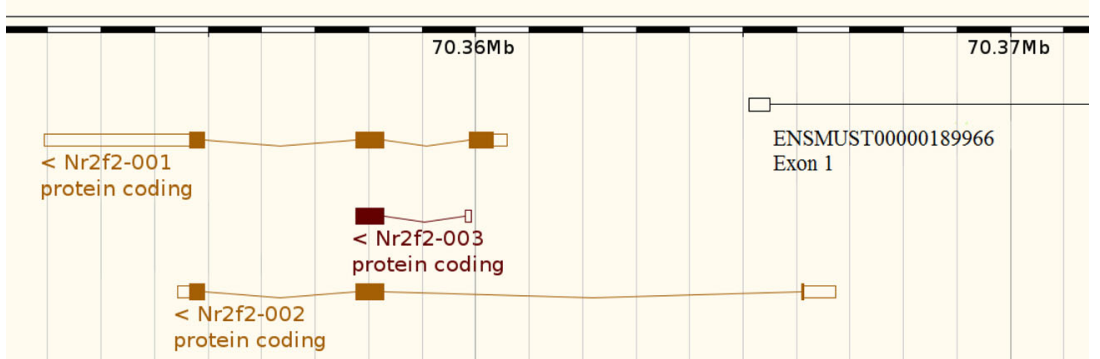

b

3T3-L1 differentiation

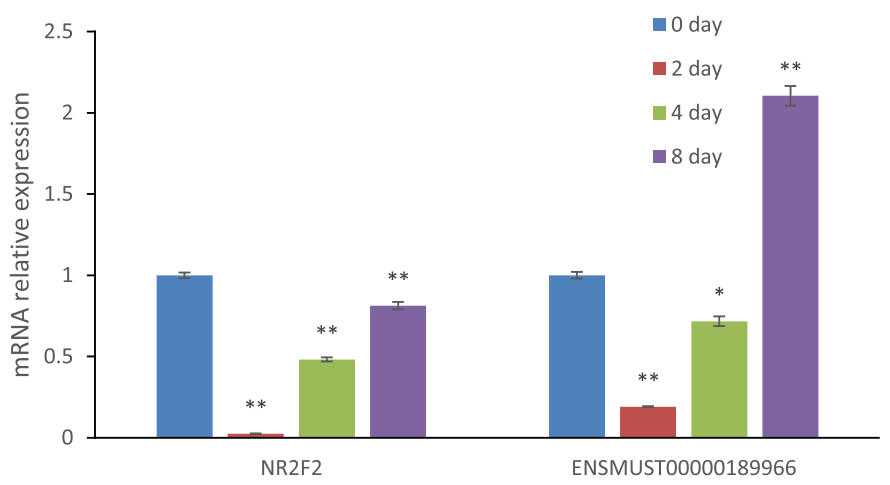

C

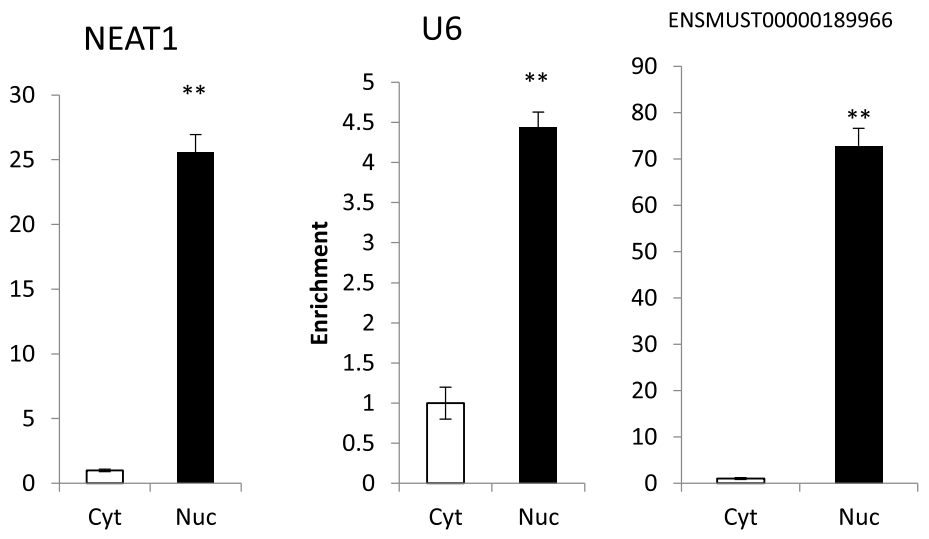

d

DHT treatment

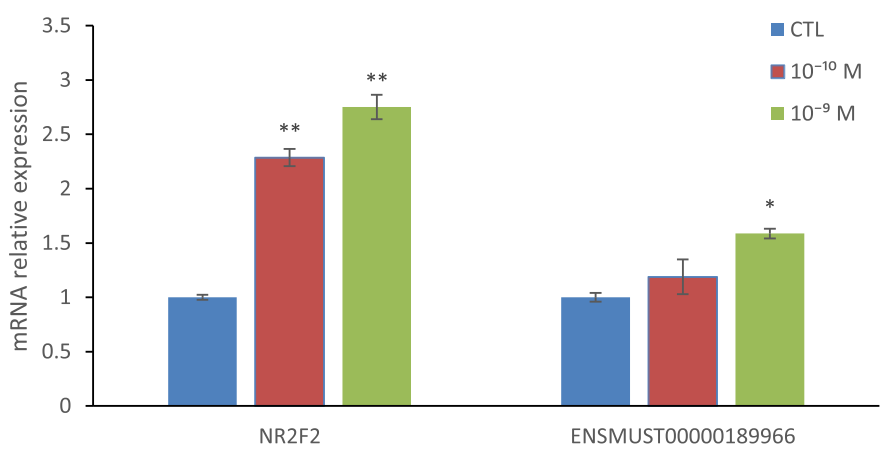

Fig. 5 (See legend on next page.) 
(See figure on previous page.)

Fig. 5 Features of NR2F2 and ENSMUST00000189966. a Gene structure of NR2F2 and ENSMUST00000189966. NR2F2-001, NR2F2-002 and NR2F2-003 are different isoforms. The scale in the top show the location in the chromosome 7. ENSMUST00000189966 exon 1 overlapped with NR2F2-002 intron 2. b Q-PCR analysis of NR2F2 and ENSMUST00000189966 gene expression during 3 T3-L1 cell culture in the differentiation medium (DM) for 0, 2, 4, and 8 days. c qPCR analyses of cytosolic (Cyt) and nuclear (Nuc) fractions. $\mathbf{d}$ The effect of dihydrotestosterone (DHT) (control (CTL), $10^{-9}$, and $10^{-10} \mathrm{M}$ ) On NR2F2 and ENSMUST00000189966 gene expression in 3 T3-L1 cells. The values represent the means \pm s.e.m., $n=3$. ${ }^{*}$ indicates $P<0.01$, *indicates $P<0.05$

found 562 recognition sites for $\mathrm{C} / \mathrm{EBP} \alpha$ in the 300 lncRNA promoter region. Sun et al. (2013) also found that $P P A R \gamma$ and $C / E B P \alpha$ were physically bound within the promoter region of adipose-enriched lncRNAs [26]. These indicated that IncRNA play an important role in lipid metabolism by interaction with key transcription factors.

Cai et al. (2014) found that 18 miRNAs were differentially expressed between intact and castrated male pigs, including miR-15a, miR-21, miR-27, miR-30, and so on [23]; Bai et al. (2014) reported that 177 miRNAs had more than 2-fold differential expression between castrated and intact male pigs, including miR-21, miR-30, miR-27, miR-103, and so on [22]. Our results were consisted with these reports, it was predicted that there were lncRNAs were the target genes for miR-21, miR30 , and miR-27. These indicated that miR-21, miR-30, and miR-27 and their target lncRNAs may play an important role in the androgen deficiency-related fat deposition, as it is widely known that miR-30a targets the androgen receptor (AR) gene [22].

The significant change after castration was the reduction of androgens, so it was predicted that $A R$ plays an important role in androgen-deficiency related fat deposition. However, there is dissension regarding the effect of castration on the $A R$ expression level: Bai et al. (2014) reported that $A R$ expression in porcine backfat dramatically decreased after castration [22]; Choi et al. (2010) reported that the expressional levels of the $A R$ gene in LD muscle were higher in bulls than steers, but in fat tissue its expression did not differ between bulls and steers [27]. Zhou et al. (2014) found that no significant difference in the $A R$ expressional level of adipose tissues between bulls and steers $(P>0.05)$. In this research, we found that castration significantly down-regulated the $A R$ level in SAD $(P<0.05)$. The effect of $A R$ on testosterone deficiency-related fat deposition needs to be further research, but we have not found lncRNA around the porcine $A R$ gene.

It was notable that ENSMUST00000189966 and its target gene $N R 2 F 2$ were significantly down-regulated after castration $(P<0.01)$. The NR2F2 gene encodes a member of the steroid thyroid hormone superfamily of nuclear receptors, and it was widely known that sex hormones belong to the steroid hormone family [28]. NR2F2 participated in the development and formation of liver and adipose tissue, and it was found that a decline of $N R 2 F 2$ led to an adipogenesis reduction [29], but $\mathrm{Xu}$ et al. (2008) reported that the knockdown of NR2F2 promoted the expression level of adipocyte marker proteins and fat deposition [30]. These two results were in stark contrast, which may be due to the fact that the differentiation stages of the 3 T3-L1 used in their research were different. Our results was consistent with Xu's research, castration down-regulated NR2F2 and ENSMUST00000189966 expression, it was inferred that serum testosterone level can affect their expression, and this effect was confirmed in 3 T3-L1 cell by adding DHT.

\section{Conclusions}

In total, 343 lncRNAs were identified in SAD from both castrated and intact male pigs, and 18 lncRNAs were found to have more than 2-fold differential expression, with $q$-values $<0.05$. The pathways analyses suggest that fatty acid synthesis and metabolism was more active in castrated pigs. In the $3 \mathrm{~T} 3-\mathrm{L} 1$ cells, the response of ENSMUST00000189966 and NR2F2 to differentiation and DHT indicated that lncRNAs with their target genes may play a regulatory role in testosterone deficiencyrelated fat deposition. We first combined sex hormones, lncRNAs, and genes together; these results provide a novel view on the role of lncRNA in testosterone deficiency-related fat deposition.

\section{Methods \\ Experimental animals}

The pigs used in this research were an indigenous Chinese breed, Huainan pigs. This breed was one of the selected superior livestock breeds in Henan province and shows heat-resistance, roughage-resistance, high litter size, and particularly high fat deposition. The pigs used in this research were reared at Henan Xing Rui Agricultural and Animal Husbandry Technology Co., LTD.

Six male Huainan pigs (three pairs of full sibs, all the pigs with similar birth weights) were used in this experiment. On day 35, for each pair of pigs, one was randomly selected to be castrated under anesthesia, and the other one was given a sham operation. All pigs were allowed access to feed and water ad libitum under normal conditions [24], and all efforts were made to minimize suffering. 


\section{Sample collections}

All the pigs were slaughtered when their body weight reached around $130 \mathrm{~kg}$ (about day 300-315 of age). Before they were slaughtered, their body weight was measured and blood samples were collected from each pig. The blood samples were centrifuged for $15 \mathrm{~min}$ at $3000 \times \mathrm{g}$ at $4{ }^{\circ} \mathrm{C}$, and then serum was collected and stored at $-80{ }^{\circ} \mathrm{C}$ [31]. The carcasses were eviscerated according to standard commercial procedures, and then the carcass weight and leaf fat weight were obtained. Back fat thickness at the $6-7$ th rib was measured using a ruler on the left side of the carcass. Subcutaneous adipose tissue samples were collected from the left side of the carcass and were rapidly frozen in liquid nitrogen, then stored at $-80{ }^{\circ} \mathrm{C}$ until analysis.

\section{Serum testosterone and TG analysis}

The serum testosterone and TG were measured using commercial enzyme-linked immunoassay kits (Beijing North Institute of Biotechnology, Beijing, China; Applygen Technologies, Beijing, China), from the absorption of $450 \mathrm{~nm}$ and $550 \mathrm{~nm}$ [32], with assay detection range were 0.05 to $20 \mathrm{ng} / \mathrm{mL}$ and 0.02 to $2 \mathrm{mM}$, respectively.

\section{Histological analysis of subcutaneous adipose tissue}

After slaughter, subcutaneous adipose tissue from six pigs were collected and fixed in $10 \%$ neutral formalin solution for $24 \mathrm{~h}$, embedded in paraffin blocks, and sectioned to a 6-um thickness. The sections were stained with hematoxylin and eosin (H\&E). Then, the slides were viewed and photographed using an Olympus Microscope (Olympus BX51, Tokyo, Japan). The mean adipocyte areas were measure using ImageJ software (NIH, Bethesda). About 50 different fields were captured for each group, 3 fields for each slide, and 5 slides for each pig.

\section{Intramuscular fat content (IMF) of the LDM and PMM}

The IMF of the LDM and PMM was measured using the soxhlet extraction method [33].

\section{LncRNA library construction and SOLiD sequencing}

Total RNA were extracted from subcutaneous adipose samples with TRIzol reagent (Invitrogen, Carlsbad, CA, USA). The RNA integrity, purity, and quality were detected using agarose gel electrophoresis, Nano-Drop ND-2000 spectrophotometer (NanoDrop products, Wilmington, USA), and Agilent 2100 Bioanalyzer (Agilent Technologies, Massy, France), respectively. The concentration of the isolated RNA was measured using a Qubit RNA BR assay for the Qubit 2.0 fluorometer. An RNA Integrity Number (RIN) value of the samples that was greater than eight could be used in sequencing.
The rRNA was removed using an epicentre RiboZero $^{\text {тм }}$ kit (Epicentre, Madison, WI, USA) from total RNA and then the RNA was fragmented (150-200 bp) with a fragmentation buffer. The double-strand cDNA was synthesized using SuperScript II reverse transcriptase (Life Techonologies, Saint Aubin, France). Then, cDNA was subjected to end-repair and phosphorylation, and subsequent purification was performed using Agencourt AMPure ${ }^{\oplus P}$ beads (Beckman Coulter, Villepinte, France). After quantitated by Agilent 2100 Bioanalyzer, the cDNA libraries were sequenced on the Illumina HiSeq 2000.

\section{Transcriptome assembly}

Clean reads was obtained by filtering the adapter and low-quality reads from the raw reads. The TopHat2 software was used to map the clean reads to the porcine reference genome (Sscrofa10.2). Transcriptomes were assembled with Cufflinks supported through Galaxy.

\section{Pipeline for the identification of IncRNA}

The lncRNA was identified by the following steps: (1) Transcripts were removed that were detected in fewer than two experiments. (2) Transcripts with single exon and with the length less than $200 \mathrm{bp}$ were removed. (3) Transcripts with coverage of less than 3 were removed; (4) Transcripts similar to or the same as known porcine small RNAs were removed. (5) Transcripts that did not pass any analyses of Coding Potential Calculator (CPC), Coding-Non-Coding-Index (CNCI), and Pfam software were removed.

\section{Prediction of target genes}

Based on the genome location of the lncRNAs and protein-coding genes, the protein-coding genes that were located upstream and downstream (within $10 \mathrm{~kb}$ and $100 \mathrm{~kb}$ ) of lncRNA were identified as target genes. The gene ontology (GO) terms of the nearest proteincoding genes with highly similar expression patterns were mapped to lncRNAs for enrichment analysis [34].

\section{Analysis of the relationship between IncRNAs with C/EBPo and miRNAs}

The binding sites of $\mathrm{C} / \mathrm{EBP} \alpha$ in the upstream 2000-bp sequence of 343 lncRNAs were analyzed using MATINSPECTOR 8.0 (Genomatix software $\mathrm{GmbH}$ ) on its default settings.

LncRNAs may act as targets of miRNAs; miRanda software was used to predict the binding sites of miRNAs in the lncRNA promoter region. We analyzed the relationship between the 343 identified IncRNAs with the 13 promoting adipogenesis miRNAs (let-7、miR-9、miR15a、 miR-17、 miR-21、miR-24、 miR-30、miR-103、miR107、miR-125b、miR-204、miR-210、 and miR-378) and 
Table 1 The effect of castration on body fatness traits and serum items in male pigs

\begin{tabular}{|c|c|c|c|}
\hline & Intact pigs ( $n=3$, Mean \pm s.e.m.) & Castrated pigs $(n=3$, Mean \pm s.e.m) & $P$-value \\
\hline \multicolumn{4}{|l|}{ Body fatness traits } \\
\hline Birth weight (kg) & $1.283 \pm 0.109$ & $1.217 \pm 0.085$ & 0.550 \\
\hline Body weight (kg) & $130.000 \pm 7.550$ & $125.667 \pm 4.841$ & 0.274 \\
\hline Carcass weight (kg) & $96.500 \pm 3.761$ & $94.266 \pm 0.545$ & 0.763 \\
\hline Leaf fat weight (kg) & $0.177 \pm 0.026$ & $1.657 \pm 0.131$ & 0.007 \\
\hline Fat percentage (\%) & $7.700 \pm 0.700$ & $26.000 \pm 2.100$ & 0.020 \\
\hline 6-7th rib fat thickness (mm) & $10.313 \pm 0.515$ & $55.407 \pm 1.241$ & 0.001 \\
\hline IMF of the LDM (\%) & $4.500 \pm 0.300$ & $5.830 \pm 0.500$ & 0.007 \\
\hline IMF of the PMM (\%) & $4.300 \pm 0.600$ & $5.240 \pm 0.900$ & 0.032 \\
\hline \multicolumn{4}{|l|}{ Serum items } \\
\hline Testosterone (ng/mL) & $11.231 \pm 0.387$ & $0.159 \pm 0.016$ & 0.001 \\
\hline Triglyceride (mM) & $0.217 \pm 0.006$ & $0.319 \pm 0.003$ & 0.008 \\
\hline Total cholesterol (mM) & $0.673 \pm 0.035$ & $1.052 \pm 0.052$ & 0.034 \\
\hline
\end{tabular}

five depressing adipogenesis miRNAs (miR-27, miR-150, miR-221, miR-222, and miR-326).

\section{Reverse transcription polymerase chain reaction and real-time PCR}

Total RNA was isolated using TRIzol reagent (Invitrogen, Carlsbad, CA, USA) according to the manufacturer's instructions. First strand cDNA was synthesized using Oligod (T)18 primer and catalyzed by PrimeScript ${ }^{\oplus}$ RT reagent Kit (Perfect Real Time) (TaKaRa, Japan). The primers used in GPCR were shown in Table 2. The reaction contained $10 \mu \mathrm{L}$ of $2 \times$ SYBR Premix Ex Taq ${ }^{\mathrm{Tm}}, 0.4 \mu \mathrm{L}$ of $50 \times$ ROX Reference Dye II, $0.5 \mu \mathrm{L}$ of $10 \mu \mathrm{M}$ forward and reverse primers, $2 \mu \mathrm{L}$ of template cDNA and $\mathrm{ddH}_{2} \mathrm{O}$, reaching a final volume of $20 \mu \mathrm{L}$. The relative lncRNA expression values were calculated using the $2^{-\Delta \Delta C t}$ method.

\section{Cell culture and differentiation}

3T3-L1 cells (Type Culture Collection of the Chinese Academy of Sciences, Shanghai, China) were maintained in the "normal medium": Dulbecco's modified Eagle's medium (DMEM) supplemented with $10 \%$ newborn calf serum (NBCS) and antibiotics (penicillin, $100 \mathrm{IU} / \mathrm{mL}$; streptomycin, $100 \mu \mathrm{g} / \mathrm{mL}$ ) at $37{ }^{\circ} \mathrm{C}$ and $5 \% \mathrm{CO}_{2}$ in a normal atmosphere incubator. Next, the medium was changed to a "maturation medium I" by adding $0.5 \mathrm{mM}$ IBMX, $1 \mathrm{mM}$ DEX, $5 \mu \mathrm{g} / \mathrm{mL}$ of insulin to the "normal medium" (day 2). After an additional $48 \mathrm{~h}$, the cell culture medium was changed to a "maturation medium II" by adding $5 \mu \mathrm{g} / \mathrm{mL}$ of insulin to the "normal medium" (day $4)$. Then the medium was changed to "normal medium" until day 14, more than $90 \%$ of the adipocyte achieved fully differentiation (indicated by the typical appearance of adipocytes). On day 14, the medium was changed to "normal medium" with or without DHT at various concentrations $\left(0,10^{-9}\right.$, and $\left.10^{-10} \mathrm{M}\right)$ for another $48 \mathrm{~h}$.

Table 2 Information of the primers used in this study

\begin{tabular}{lllll}
\hline Loci & Primer senquence & & $\begin{array}{l}\text { Size } \\
(\mathrm{bp})\end{array}$ & $\begin{array}{l}\text { Primer Efficiency } \\
(\mathrm{mean} \pm \text { SE) }\end{array}$ \\
\cline { 2 - 5 } TCONS_00311264 & Forward (5'-3') & Reverse (5'-3') & 224 & $0.95 \pm 0.01$ \\
TCONS_00392681 & TGTGGAGGAAACGAGGGT & GAAAGATGCCGCTTGGA & 160 & $0.97 \pm 0.04$ \\
TCONS_01199263 & TATTCTGGGCAGGATTG & GCAGGTCTTTCGAGGTA & 228 & $0.84 \pm 0.04$ \\
TCONS_01743394 & CTCGCTCAACTCAGCATTC & TGGACAAGGGTTAGAAGTAAAT & 187 & $0.88 \pm 0.02$ \\
TCONS_00465264 & TATTGAAATGCGTGAGGCT & CTGGAACGAGCAAGGAGA & 121 & $0.86 \pm 0.02$ \\
TCONS_00625772 & GCTGCGCTCTATTCTGTG & GTATGCCAGGAGGGAGGA & 122 & $0.94 \pm 0.06$ \\
TCONS_00869992 & GGCTTCCGCCTCGTGAT & CTGGCTGCCTTCGTTCC & 174 & $0.92 \pm 0.03$ \\
TCONS_01520466 & AGGGAGAAGGAGTGAGACAAG & GGGAAGGCAGATGGACAA & 158 & $0.87 \pm 0.05$ \\
NR2F2 & TTTCTTGGGTAAACATCTGG & GTGACCTTGTGGGCAGT & 190 & $0.94 \pm 0.04$ \\
ENSMUSG00000100005 & GGACCACATACGGATCTTC & CTGGCTCCTAACGTACTCTT & 296 & $0.95 \pm 0.02$ \\
\hline
\end{tabular}




\section{Isolation of RNA from nuclear and cytoplasmic}

3T3-L1 cells, cultured with differentiation medium in a T-75 flask, were harvested after which achieved fully differentiation. The harvested cells were washed once by using cold PBS. At $4{ }^{\circ} \mathrm{C}$, these cells were centrifuged at $500 \times \mathrm{g}$ for $3 \mathrm{~min}$. The nuclear and cytoplasmic RNA were isolated using a PARIS ${ }^{\mathrm{Tm}}$ kit (Life Technologies, USA). The RNA from nuclear and cytoplasmic was reverse transcribed with the equal amount. The primers for detecting the distribution of U6 and NEAT1 genes were modeled after Zhao et al. (2015) [35].

\section{Statistical analysis}

The results are reported as the mean \pm standard error mean (s.e.m.). Data analyses were performed using the statistical $R$ package.

\section{Additional files}

Additional file 1: The sequences of identified IncRNAs in FASTA format. (SEQ 514 kb)

Additional file 2: Table S1. The binding sites of CEBPa at identified IncRNAs. (XLSX $27 \mathrm{~kb}$ )

Additional file 3: Table S2. Adipogenesis related miRNAs binding sites at identified IncRNAs. (XLSX 66 kb)

Additional file 4: Figure S1. Comparison of features of porcine IncRNAs and mRNAs. Note: Comparison of the lengths (a), ORF lengths (b) and exon numbers (c) of porcine IncRNAs and mRNAs. (DOCX $302 \mathrm{~kb}$ )

Additional file 5: Table S3. The differentially expressed IncRNA between castrated and intact male pigs and their distribution. (XLSX 9 kb)

Additional file 6: Table S4. The differentially expressed mRNA between castrated and intact male pigs and their distribution. (XLSX $222 \mathrm{~kb}$ )

Additional file 7: Figure S2. Verification of gene expression analysis by quantitative realtime PCR (qRT-PCR). Note: Individual gene expression ratios were calculated using foldchange generated by RNA-seq and plotted against calculations done for the same gene using qRT-PCR. (DOCX $17 \mathrm{~kb}$ )

\section{Abbreviations}

AMPK: AMP-activated protein kinase; C/EBPa: CCAAT/enhancer binding protein alpha; $\mathrm{CNCl}$ : Coding-Non-Coding-Index; CPC: Coding Potential Calculator; DHT: Dihydrotestosterone; DMEM: Dulbecco's modified Eagle's medium; FPKM: Fragments per kilobase of exons per million fragments mapped; GO: Gene ontology; H\&E: Hematoxylin and eosin; LDM: Longissimus dorsi muscle; lincRNAs: Intergenic IncRNAs; IncRNAs: Long non-coding RNAs; NBCS: Newborn calf serum; NR2F2: Nuclear Receptor Subfamily 2 Group F Member 2; ORF: Open reading frame; PMM: Psoas major muscle; PPARY: Peroxisome proliferator-activated receptor gamma; RIN: RNA Integrity Number; SAD: Subcutaneous adipose tissue; TG: Triglyceride

\section{Acknowledgements}

Not applicable.

\section{Funding}

This study was supported by the National Natural Science Foundation of China (31601927), the Henan Province Foundation and Advanced Technology Research Project (162300410143), and the Dr. Start-up Fund Project in Henan Academy of Agricultural Science.

\section{Availability of data and materials}

All the basic data series were submitted to National Center for Biotechnology Information Sequence Read Archive with accession number SRX1712506 and SRX1712510.

\section{Authors' contributions}

JW, JFC, and CJL designed the experiments. JW, JQZ, and BWG gathered the samples. JW performed the data analysis, interpreted the results, and drafted the manuscript. LSH, ZHS, YG, and XXB participated in intellectual discussions. BSX and WDS provided guidance on the experimental design and funding. All authors read and approved of the final manuscript.

\section{Ethics approval}

The methods were performed in accordance with the guidelines of the Good Experimental Practices adopted by the Institute of Animal Science. And all experimental protocols were approved by the Institute of Animal Science of the Chinese Academy of Agricultural Sciences.

\section{Consent for publication}

Not applicable.

\section{Competing interests}

The authors declare that they have no competing interests.

\section{Publisher's Note}

Springer Nature remains neutral with regard to jurisdictional claims in published maps and institutional affiliations.

\section{Author details}

${ }^{1}$ Henan Key Laboratory of Farm Animal Breeding and Nutritional Regulation, Institute of Animal Husbandry and Veterinary Science, Henan Academy of Agricultural Sciences, No.116 Huayuan road, Zhengzhou 450002, People's Republic of China. ${ }^{2}$ United States Department of Agriculture-Agricultural Research Service, Bovine Functional Genomics Laboratory, Beltsville, MD 20705, USA. ${ }^{3}$ Xinxian Bureau of Animal Husbandry, Xinxian, 465550 Beijing, People's Republic of China.

Received: 13 June 2016 Accepted: 27 June 2017

Published online: 19 July 2017

\section{References}

1. Feinberg AP, Irizarry RA, Fradin D, Aryee MJ, Murakami P, Aspelund T et al. Personalized epigenomic signatures that are stable over time and covary with body mass index. Science translational medicine. 2010; 2(49):49ra67.

2. Miljkovic I, Cauley JA, Dressen AS, Gordon CL, Goodpaster BH, Kuller LH, et al. Bioactive androgens and glucuronidated androgen metabolites are associated with subcutaneous and ectopic skeletal muscle adiposity among older black men. Metabolism. 2011;60(8):1178-85.

3. Wolf E, Braun-Reichhart C, Streckel E, Renner S. Genetically engineered pig models for diabetes research. Transgenic Res. 2014;23(1):27-38.

4. Gispert M, Angels Oliver M, Velarde A, Suarez P, Perez J, Font i Furnols M. Carcass and meat quality characteristics of immunocastrated male, surgically castrated male, entire male and female pigs. Meat Sci. 2010;85(4):664-70.

5. Saintilan R, Sellier P, Billon Y, Gilbert H. Genetic correlations between males, females and castrates for residual feed intake, feed conversion ratio, growth rate and carcass composition traits in large white growing pigs. J Anim Breed Genet. 2012;129(2):103-6.

6. Zhao J, Sun BK, Erwin JA, Song JJ, Lee JT. Polycomb proteins targeted by a short repeat RNA to the mouse X chromosome. Science. 2008; 322(5902):750-6.

7. Ramos AD, Andersen RE, Liu SJ, Nowakowski TJ, Hong SJ, Gertz CC, et al. The long noncoding RNA Pnky regulates neuronal differentiation of embryonic and postnatal neural stem cells. Cell Stem Cell. 2015;16(4): 439-47.

8. Rosa A, Ballarino M. Long noncoding RNA regulation of pluripotency. Stem Cells Int. 2016;2016:1797692.

9. Xiao T, Liu L, Li H, Sun Y, Luo H, Li T, et al. Long noncoding RNA ADINR regulates Adipogenesis by transcriptionally activating C/EBPalpha. Stem cell reports. 2015;5(5):856-65.

10. Zhao XY, Li S, Wang GX, Yu Q, Lin JD. A long noncoding RNA transcriptional regulatory circuit drives thermogenic adipocyte differentiation. Mol Cell. 2014;55(3):372-82.

11. Wang F, Tong Q. Transcription factor PU.1 is expressed in white adipose and inhibits adipocyte differentiation. American journal of physiology Cell physiology. 2008;295(1):C213-20. 
12. Pang WJ, Lin $L G$, Xiong $Y$, Wei $N$, Wang $Y$, Shen $Q$ W, et al. Knockdown of PU.1 AS IncRNA inhibits adipogenesis through enhancing PU.1 mRNA translation. J Cell Biochem. 2013;114(11):2500-12.

13. Li M, Sun X, Cai H, Sun Y, Plath M, Li C, et al. Long non-coding RNA ADNCR suppresses adipogenic differentiation by targeting miR-204. Biochim Biophys Acta. 2016;1859(7):871-82.

14. Cabili MN, Trapnell C, Goff L, Koziol M, Tazon-Vega B, Regev A, et al. Integrative annotation of human large intergenic noncoding RNAs reveals global properties and specific subclasses. Genes Dev. 2011;25(18):1915-27.

15. Traish AM, Zitzmann M. The complex and multifactorial relationship between testosterone deficiency (TD), obesity and vascular disease. Rev Endocr Metab Disord. 2015;16(3):249-68.

16. Wang X, Zhu H, Snieder H, Su S, Munn D, Harshfield G, et al. Obesity related methylation changes in DNA of peripheral blood leukocytes. BMC Med. 2010;8:87.

17. Estep JM, Goodman Z, Sharma H, Younossi E, Elarainy H, Baranova A, et al. Adipocytokine expression associated with miRNA regulation and diagnosis of NASH in obese patients with NAFLD. Liver Int. 2015;35(4):1367-72.

18. Van Campenhout J, Vanreusel A, Van Belleghem S, Derycke S. Transcription, signaling receptor activity, oxidative phosphorylation, and fatty acid metabolism mediate the presence of closely related species in distinct intertidal and cold-seep habitats. Genome Biol Evol. 2016;8(1):51-69.

19. Tepaamorndech S, Kirschke CP, Pedersen TL, Keyes WR, Newman JW, Huang L. Zinc transporter 7 deficiency affects lipid synthesis in adipocytes by inhibiting insulin-dependent Akt activation and glucose uptake. FEBS J. 2016;283(2):378-94.

20. Rao Y, Liu H, Gao L, Yu H, Ou TM, Tan JH, et al. Synthesis and biological evaluation of novel Bouchardatine derivatives as Potential Adipogenesis/ lipogenesis inhibitors for Antiobesity treatment. J Med Chem. 2015;58(23): 9395-413.

21. Le Guevel R, Oger F, Martinez-Jimenez CP, Bizot M, Gheeraert C, Firmin F, et al. Inactivation of the Nuclear Orphan Receptor COUP-TFIl by Small Chemicals. 2017;12(3):654-63.

22. Bai Y, Huang JM, Liu G, Zhang JB, Wang JY, Liu CK, et al. A comprehensive microRNA expression profile of the backfat tissue from castrated and intact full-sib pair male pigs. BMC Genomics. 2014;15:47.

23. Cai Z, Zhang L, Chen M, Jiang X, Xu N. Castration-induced changes in microRNA expression profiles in subcutaneous adipose tissue of male pigs. J Appl Genet. 2014;55(2):259-66.

24. Li M, Wu H, Luo Z, Xia Y, Guan J, Wang T, et al. An atlas of DNA methylomes in porcine adipose and muscle tissues. Nat Commun. 2012;3:850.

25. Esteller M. Epigenetics in cancer. N Engl J Med. 2008;358(11):1148-59.

26. Lavebratt $C$, Almgren M, Ekstrom TJ. Epigenetic regulation in obesity. International journal of obesity (2005). 2012; 36(6):757-65.

27. Musri MM, Gomis R, Parrizas M. Chromatin and chromatin-modifying proteins in adipogenesis. Biochemistry and cell biology =. Biochimie et biologie cellulaire. 2007;85(4):397-410.

28. Pereira FA, Tsai MJ, Tsai SY. COUP-TF orphan nuclear receptors in development and differentiation. Cell Mol Life Sci. 2000;57(10):1388-98.

29. Jeong BC, Kang $\mathrm{H}$, Hwang YC, Kim SH, Koh JT. MicroRNA-194 reciprocally stimulates osteogenesis and inhibits adipogenesis via regulating COUP-TFII expression. Cell Death Dis. 2014;5:e1532.

30. Xu Z, Yu S, Hsu CH, Eguchi J, Rosen ED. The orphan nuclear receptor chicken ovalbumin upstream promoter-transcription factor II is a critical regulator of adipogenesis. Proc Natl Acad Sci U S A. 2008;105(7):2421-6.

31. Cai Z, Xi H, Pan Y, Jiang X, Chen $L$, Cai $Y$, et al. Effect of testosterone deficiency on cholesterol metabolism in pigs fed a high-fat and highcholesterol diet. Lipids Health Dis. 2015;14:18.

32. Deng J, Liu S, Zou L, Xu C, Geng B, Xu G. Lipolysis response to endoplasmic reticulum stress in adipose cells. J Biol Chem. 2012;287(9):6240-9.

33. Hawthorne SB, Grabanski CB, Martin E, Miller DJ. Comparisons of soxhlet extraction, pressurized liquid extraction, supercritical fluid extraction and subcritical water extraction for environmental solids: recovery, selectivity and effects on sample matrix. J Chromatogr A. 2000;892(1-2):421-33.

34. Pauli A, Valen E, Lin MF, Garber M, Vastenhouw NL, Levin JZ, et al. Systematic identification of long noncoding RNAs expressed during zebrafish embryogenesis. Genome Res. 2012;22(3):577-91.

35. Zhao W, Mu Y, Ma L, Wang C, Tang Z, Yang S, et al. Systematic identification and characterization of long intergenic non-coding RNAs in fetal porcine skeletal muscle development. Sci Rep. 2015;5:8957.

\section{Submit your next manuscript to BioMed Central and we will help you at every step:}

- We accept pre-submission inquiries

- Our selector tool helps you to find the most relevant journal

- We provide round the clock customer support

- Convenient online submission

- Thorough peer review

- Inclusion in PubMed and all major indexing services

- Maximum visibility for your research

Submit your manuscript at www.biomedcentral.com/submit
Biomed Central 